\title{
Originales
}

\section{Análisis bibliométrico y temático de la producción científica sobre salud laboral relacionada con nutrición, alimentación y dieta, indexada en MEDLINE}

\section{Bibliometric and Thematic Analysis of Scientific Production on Occupational Health Related to Nutrition, Food and Diet, indexed in MEDLINE}

\author{
Liliana Melián-Fleitas ${ }^{1,2}$, Álvaro Moisés Franco-Pérez', Javier Sanz Valero²,3
}

1. Servicio Canario de Salud. Atención Primaria de Lanzarote. Lanzarote. España.

2. Universidad de Alicante. Departamento de Enfermería Comunitaria, Medicina Preventiva y Salud Pública e Historia de la Ciencia. Alicante. España.

3. Universidad Miguel Hernández. Departamento de Salud Pública e Historia de la Ciencia. San Juan de Alicante. España.

Recibido: 30-11-2018

Aceptado: 31-01-2019

\author{
Correspondencia \\ Liliana Melián-Fleitas \\ Servicio Canario de Salud \\ Atención Primaria de Lanzarote. Lanzarote. España \\ Correo electrónico: lilianamelian@hotmail.es
}

Resumen

Objetivo: Análisis bibliométrico y temático de la producción científica sobre salud laboral relacionada con la nutrición, alimentación y dieta indexada en MEDLINE.

Método: Estudio descriptivo transversal. Los datos se obtuvieron de la base de datos MEDLINE (PubMed). La ecuación de búsqueda fue "Diet, Food, and Nutrition"[Mesh] AND "Occupational Health"[Mesh] AND "HUMANS"[MeSH Terms]. Se analizaron los principales indicadores bibliométricos. Se segmentó la búsqueda por épocas (desde el año 1949 hasta 2008 incluido, primera época y desde 2009 a 2017 incluido, segunda época). La fecha de la búsqueda fue febrero 2018.

Resultados: Se analizaron 386 referencias. El número de originales fue de 229 (59,3\%), identificando 248 instituciones, con Índice de colaboración de 4,0 $\pm 2,9$ autores/artículo y máximo de 16, siendo 2015 el año de mayor producción. La edad media de los documentos fue de 15,6 años y el Índice de Price 28,5\%. La distribución geográfica fue mayoritariamente estadounidense (EE. UU.), con 115 trabajos, y el idioma predominante el inglés con 286 artículos. Se recuperaron 233 revistas y se pudo acceder de forma gratuita a 126 artículos. Hubo diferencias significativas entre los indicadores analizados por épocas.

Conclusiones: Existencia de relativa obsolescencia, orientación anglófona y filiación estadounidense. Colaboración institucional limitada y destacada de autores. Amplio acceso gratuito al documento primario e impacto irregular de las publicaciones. Notable mejoría de los indicadores bibliométricos a partir del 2008.

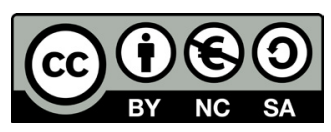

https://creativecommons.org/licenses/by-nc-sa/4.0/ 
La clasificación temática cumplió con la materia investigada. La temática está aún en desarrollo por los potenciales beneficios para trabajadores, empresas y gobiernos.

Med Segur Trab (Internet). 2019;65(254):10-23

Palabras clave: Salud Laboral; Nutrición, Alimentación y Dieta; Bibliometría; Indicadores Bibliométricos; Descriptores de Ciencias de la Salud.

Abstract

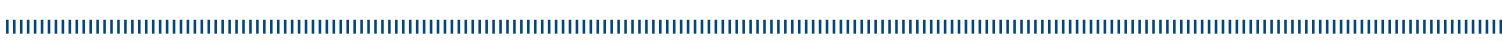

Objective: Bibliometric and thematic analysis of scientific production on occupational health related to nutrition, food and diet indexed in MEDLINE.

Metbod: Cross-sectional study. The data were obtained from the MEDLINE database (PubMed). The search equation was "Diet, Food, and Nutrition"[Mesh] AND "Occupational Health"[Mesh] AND "HUMANS"[MeSH Terms]. The main bibliometric indicators were analysed. The search was segmented by epochs (first period from the year 1949 to 2008 and second period from 2009 to 2017). Search date: February 2018.

Results: 386 references were analysed. The original number was 229 (59.3\%), identifying 248 institutions and collaboration index of $4.0 \pm 2.9$ authors / article, being 16 the maximum and 2015 the year of highest production. The average of documents was 15.6 years and the Price Index 28.5\%. The United States of America was the main geographical distribution area with 115 works, being English the dominant language in 286 articles. 233 journals were retrieved. There was free access to 126 articles. There were significant differences between the indicators analysed by epochs.

Conclusions: Existence of relative obsolescence, Anglophone orientation and American affiliation. It is an institutional author collaboration which is limited and outstanding, with wide free access to the primary document and irregular impact publications. The bibliometric indicators were clearly improved from the year 2008. The thematic classification complied with the researched subject. The thematic is still under development due to the potential benefits for workers, companies and governments.

Med Segur Trab (Internet). 2019;65(254):10-23

Keywords: Occupational Health; Diet, Food, and Nutrition; Bibliometrics; Bibliometric Indicators; Medical Subject Headings. 


\section{INTRODUCCIÓN}

Bernardino Ramazzini (1633-1714), en su libro sobre enfermedades profesionales e higiene industrial, publicado en 1700 con el título de "Tratado de las enfermedades de los artesanos", denunció las condiciones insalubres de los mineros, las intoxicaciones con plomo y mercurio en diferentes profesiones, los peligros del sedentarismo y la influencia de los factores ambientales. Recomendaba medidas preventivas que debían abarcar tres niveles: la eliminación del riesgo, su control a través de la ingeniería y la prevención personal $^{1}$.

Ramazzini (considerado el padre de la Medicina del Trabajo) fue un adelantado a su época al reclamar la reducción de la jornada laboral, implementación de descansos, la adopción de posturas correctas (lo que hoy llamamos ergonomía), la adecuada alimentación y la lucha contra la pobreza ${ }^{1}$.

Actualmente la salud comunitaria estudia la influencia de la adecuada alimentación/ nutrición (dieta alimentaria) en la salud laboral y como acometer sus limitaciones (desnutrición) y sus excesos (obesidad). En este sentido, el National Institute for Occupational Safety and Health (NIOSH), que forma parte de los Centers for Disease Control and Prevention de los EE. UU., tiene como objetivo concienciar a los empleadores y empoderar a los trabajadores para crear lugares de trabajo seguros y saludables. Una de sus líneas maestras es la "Total Worker Health (TWH)", una estrategia que integra la seguridad ocupacional y la protección de la salud a fin de prevenir lesiones y enfermedades de los trabajadores y mejorar su salud y bienestar, siendo uno de los temas relevantes el acceso a alimentos saludables y económicos ${ }^{2}$.

Ahora bien, si ya existe un buen número de documentación acerca de la salud laboral relacionada con la nutrición, alimentación y dieta, no existen investigaciones que informen sobre la producción científica de esta documentación y el impacto que supone en el mundo académico y social ${ }^{3}$.

En consecuencia, dado el interés que suscita un campo de estudio determinado, como es el caso de la salud laboral relacionada con la nutrición, alimentación y dieta, es útil conocer la progresión de su investigación y del conocimiento creado. Así como, estudiar de forma retrospectiva los resultados generados y como se han dado a conocer ${ }^{4,5}$.

Este análisis documental permitirá conocer el aumento y desarrollo de la disciplina a estudio y ver el potencial investigador de los grupos e instituciones involucradas. En este sentido, los estudios métricos han alcanzado gran importancia en la política científica y de gestión, dado el auge de la cultura de evaluación y rendición de cuentas, en la medida que el conocimiento científico es visto como un valor estratégico (generación de outputs) ${ }^{6}$. Los estudios bibliométricos tienen por objeto el tratamiento y análisis cuantitativo de las publicaciones científicas, constituyendo en la actualidad la herramienta esencial para el conocimiento de la actividad investigadora, aportando datos sobre la situación científica de un país o tema de investigación, permitiendo evaluar el rendimiento de la actividad científica y su impacto en la comunidad ${ }^{7}$.

Por todo ello, el objetivo de este trabajo fue conocer y evaluar, mediante el análisis documental, la actividad científica, la producción, el consumo y el impacto de las publicaciones científicas, indizadas en MEDLINE, sobre la salud laboral relacionada con la nutrición, alimentación y dieta.

\section{MATERIAL Y MÉTODO}

\section{Diseño}

Estudio descriptivo transversal de la producción científica sobre salud laboral relacionada con la nutrición, alimentación y dieta. 


\section{Fuente de obtención de los datos}

Se obtuvieron de la consulta directa y acceso, vía Internet, a la documentación científica existente en la base de datos MEDLINE, vía PubMed.

\section{Unidad de análisis}

Artículos sobre salud laboral relacionada con la nutrición, alimentación y dieta indizados en la base de datos bibliográfica indicada.

\section{Búsqueda bibliográfica}

Para construir la ecuación de búsqueda se consultó el Medical Subject Headings (MeSH), Thesaurus desarrollado por la U.S. National Library of Medicine para conocer los Descriptores adecuados. Esta ecuación se desarrolló mediante la intersección booleana de dos Descriptores y un límite:

"Diet, Food, and Nutrition"[Mesh] AND "Occupational Health"[Mesh] AND "HUMANS"[MeSH Terms]

\section{Población a estudio}

El tamaño muestral se calculó mediante la estimación de parámetros poblacionales para una población infinita, (valor esperado $=0,5$; precisión del intervalo $=0,05$; nivel de confianza $=0,95)$.

Para la selección de las referencias se efectuó un muestreo aleatorio simple sin reemplazo, tomando como base el número total de referencias bibliográficas obtenidas.

\section{Indicadores a estudio}

- Producción científica, calculada según número de artículos indizados.

- Tipología documental: Características indicativas que distinguen al documento.

- Número de artículos citables (suma de artículos de investigación original y de revisión).

— Índice de productividad: logaritmo del número de trabajos originales publicados.

- Año de publicación.

— Edad: Año 2018 menos año de publicación del artículo.

- Autoría: número de autores que firman el documento.

- Índice de colaboración (cociente entre el número de firmas y el número de trabajos).

- Semiperiodo de Burton-Kebler (Obsolescencia/actualidad): Mediana de la distribución del conjunto de las referencias ordenadas por su antigüedad.

- Índice de Price: porcentaje de referencias con edad menor de 5 años.

- Idioma: lengua de publicación del artículo.

- Distribución geográfica de procedencia de los artículos.

- Índice de colaboración institucional: cociente entre el número de centros firmantes y el número de artículos.

- Filiación institucional del primer firmante e Índice de Lotka. Las instituciones se agruparon en tres niveles de rendimiento: pequeños productores (Índice de transitoriedad = un único trabajo), medianos productores (entre 2 y 9 trabajos) y grandes productores (10 o más trabajos).

- Revista: nombre completo de la revista donde se ha publicado el artículo.

- Impacto de las publicaciones según el Journal Citation Report Science Edition Database (JCR) y del SCImago Journal Rank (SJR). 
- Dispersión: núcleo principal de Bradford (conjunto de revistas de mayor pertinencia para un área de conocimiento).

— Enlace: Existencia de enlace al documento desde PubMed.

- Acceso: Posibilidad de acceder gratuitamente al texto completo del artículo a través del enlace existente en PubMed.

- Indización mayor: Descriptores (MeSH), como Major Topic, que representan el contenido principal de cada artículo.

- Área temática: clasificación de los artículos según las 16 grandes áreas temáticas del Thesaurus MeSH primer nivel.

- Digital Object Identifier (DOI): Presencia de este identificador único que permite un acceso rápido al documento primario.

\section{Análisis de los datos}

Las variables cualitativas se describieron por su frecuencia y porcentaje, las cuantitativas mediante su Media y Desviación Estándar; representando las más relevantes mediante la utilización de tablas y gráficos. Se utilizó la Mediana (Me) y la Moda como medida de tendencia central, con la correspondiente amplitud intercuartílica (AIQ). Para conocer la evolución de algunos indicadores se segmentó el período de estudio en dos etapas, tomando como punto de corte el Índice de Burton Kebler (la Mediana). $1^{\text {a }}$ época:

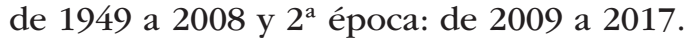

El crecimiento de la producción científica se examinó mediante el análisis de regresión. La existencia de asociación entre variables cualitativas se analizó mediante la prueba de Chi-cuadrado de Pearson. Para comprobar la significación en la diferencia de medias para muestras independientes se utilizó la prueba t de Student. El nivel de significación utilizado en todos los contrastes de hipótesis fue $\alpha \leq 0,05$, describiéndose los Intervalos de Confianza al 95\%.

Para el cálculo del tamaño muestral y la selección de las referencias del muestreo se utilizó el programa EPIDAT versión 4.2. Para el almacenamiento y análisis estadístico se usó el programa IBM-SPSS, versión 22 para Windows.

El control de la calidad de la información se efectuó mediante la doble entrada de los datos, corrigiendo las inconsistencias mediante la consulta con los datos originales.

\section{RESULTADOS}

\section{Producción científica}

De la búsqueda realizada en la base de datos MEDLINE se obtuvo un total de 609 referencias. Tras el cálculo del tamaño muestral se analizaron 386 documentos.

El primer artículo indizado fue publicado en "Arbeitsphysiologie; internationale Zeitschrift für angewandte Physiologie» en 1949, siendo el 2015 el año de mayor producción sobre la temática a estudio con 33 artículos, un 8,6\% del total (IC 95\% 5,8 11,3).

La relación entre el número de publicaciones por año muestra que el modelo de regresión con un mayor ajuste fue el lineal $(\mathrm{p}<0,001)$, con tendencia creciente, siendo el coeficiente de determinación $\mathrm{R}^{2}=46,0$; ver figura 1 . 
Figura 1. Evolución de la producción científica sobre salud laboral, nutrición y dieta, recuperada de la base de datos MEDLINE en febrero de 2018, y ajuste al modelo lineal.

35

30

\section{Obsolescencia/actualidad de la producción científica}

La edad media de los documentos analizados fue 15,6 \pm 18,4 años, con Mediana de 9 y AIQ de 14, con Máximo de 68 años; ver figura 1. El Índice de Price (IP) obtenido fue del 28,5\% (IC95\% 24,0 - 33,0).

\section{Tipología documental}

La tipología documental más frecuente fue el artículo original con 229 documentos (59,3\%; IC95\% 54,4 - 64,2), siendo el índice de productividad de 2,4. El número revisiones fue de 40 (10,4\%; IC95\% 7,3 - 13,4) y el de ensayos clínicos de 29 (7,5\%; IC95\% 4,910,1).

La frecuencia y porcentaje de los artículos citables (originales + revisiones), computables para el cálculo del impacto, fue de 269 (69,7\%; IC95\% 65,1 - 74,3).

Se observaron diferencias significativas, relacionadas con la tipología documental, entre las dos épocas a estudio (Chi-cuadrado de Pearson $=74,8 ; \mathrm{gl}=9 ; \mathrm{p}<0,001$ ).

\section{Procedencia geográfica y filiación institucional}

La distribución geográfica de las referencias analizadas fue mayoritariamente estadounidense, con aproximadamente un tercio de la producción: 115 trabajos (29,8\%; IC95\% 25,2 - 34,4). En segundo lugar se situó Japón con 23 artículos (6,0\%; IC95\% 3,6 8,3) seguido de Reino Unido con 17 (4,4\%; IC95\% 2,4 - 6,5), Brasil con 14 (3,6\%; IC95\% 1,8 - 5,5), Italia con 12 (3,1\%; IC95\% 1,4 - 4,8), Australia con 11 (2,9\%; IC95\% 1,2 - 4,5) y Holanda con 10 (2,6\%; IC95\% 1,0 - 4,2). El resto de países presentaron una frecuencia menor de 10. En 91 artículos (23,6\%; IC95\% 19,3 - 27,8) no constaba el país y los documentos con filiación española fueron 5 (1,3\%; IC95\% 0,2 - 2,4).

En el conjunto de la búsqueda se identificaron documentos de 46 nacionalidades distintas. Por continentes se observa la preponderancia de América del Norte con 123 publicaciones (31,9\%; IC95\% 27,2 - 36,5), seguida de Europa con 82 (21,2\%; IC95\% 17,2 - 25,3), Asia con 50 (12,9\%; IC95\% 9,6 - 16,3), América del Sur con 17 (4,4\%; IC95\% 2,4 - 6,4), Oceanía con 13 (3,4\%; IC95\% 1,6 - 5,2) y África con 10 (2,6\%; IC95\% $1,0-4,2)$.

Los países con 10 o más publicaciones, ordenados según la época, pueden consultarse en la tabla I, observándose diferencias significativas entre las mismas (Chi-cuadrado de Pearson $=111,5 ; \mathrm{gl}=45 ; \mathrm{p}<0,001)$. 
Tabla I. Países con 10 o más publicaciones según época a estudio.

\begin{tabular}{|c|c|c|c|c|}
\hline & País & $1^{a}$ época $(1949-2008)$ & $2^{a}$ época $(2009-2017)$ & Total \\
\hline & EE.UU & 51 & 64 & 115 \\
\hline & Japón & 10 & 13 & 23 \\
\hline $\begin{array}{l}\mathbb{N} \\
\boldsymbol{Z} \mathbb{N}\end{array}$ & Reino Unido & 7 & 10 & 17 \\
\hline & Brasil & 3 & 11 & 14 \\
\hline & Italia & 3 & 9 & 12 \\
\hline & Australia & 2 & 9 & 11 \\
\hline & Holanda & 4 & 6 & 10 \\
\hline
\end{tabular}

Se observó colaboración entre países en 13 de los trabajos analizados (3,4\%; IC95\% 1,6 - 5,2). La Media fue de 0,8 \pm 0,8, Mediana de 1, Mínimo de 0, Máximo de 11 y AIQ de 0 .

Se identificaron trabajos publicados de 248 instituciones, con una Mediana de 1 centro por artículo y AIQ de 0,25; Máximo de 12 y Media -Índice de colaboración institucional- $1,1 \pm 1,4$.

La colaboración institucional fue favorable a la $2^{\text {a }}$ época $(1,6$ frente a 0,6$)$, observándose diferencias significativas en las medias del Índice de colaboración institucional entre ambas épocas ( $\mathrm{t}$ de Student $=-7,1 ; \mathrm{gl}=265,2 ; \mathrm{p}<0,001$ ).

Un total de 47 estudios (12,2\%; IC95\% 8,9 - 15,4) fueron realizados en colaboración entre 2 o más centros. La filiación de los documentos analizados se clasificó, extrapolando el Índice de Lotka, en tres niveles de rendimiento: pequeños productores, o Índice de Transitoriedad (un único trabajo), donde encontramos 243 centros (63,0\%; IC95\% 58,1 $67,8)$; medianos productores (entre 2 y 9 trabajos) con 44 centros $(11,4 \%$; IC95\% 8,2 14,6); y grandes productores (10 o más trabajos) con 3 centros $(0,8 \%$; IC95\% 0,00 - 1,7). En 96 artículos (24,9\%; IC95\% 20,6 - 29,2) no figuraba filiación alguna.

\section{Idioma de publicación}

El principal idioma de publicación fue el inglés con 286 artículos (74,1\%; IC95\% 69,7 - 78,5), seguido del ruso con 27 (7,0\%; IC95\% 4,5 - 9,5), el francés y portugués con 9 (2,3\%; IC95\% 0,8 - 3,8), el japonés con 8 (2,1\%; IC95\% 0,7 - 3,5) y el español, italiano, alemán y sueco con 5 (1,3\%; IC95\% 0,2 - 2,4). El resto de idiomas tuvo una frecuencia inferior a 5. En 20 documentos (5,2\%; IC95\% 3,0 - 7,4) no constaba el idioma de publicación.

Se observaron diferencias significativas, en relación al idioma, entre las épocas a estudio (Chi-cuadrado de Pearson $=61,0 ; \mathrm{gl}=14 ; \mathrm{p}<0,001$ ) (Tabla II).

\section{Autoría}

Se contabilizaron un total de 1556 firmantes, con un Máximo de 16 autores observado en 2 artículos (0,5\%; IC95\% 0,0 - 1,2). La Media de autores -Índice de colaboración- fue de 4,0 $\pm 2,9$. La Mediana fue de 3 autores por artículo y la Moda de 1 autor. La AIQ fue de 4. Se observaron diferencias significativas entre las medias de autores/artículo de las dos épocas a estudio: 3,3 versus 4,9 (t de Student $=-5,5 ;$ gl= 384 $\mathrm{p}<0,001$ ); ver figura 2 . 
Tabla II. Idiomas de publicación divididos según época a estudio.

\begin{tabular}{|c|c|c|c|c|}
\hline & Idioma & $1^{a}$ época (1949 - 2008) & $2^{a}$ época $(2009-2017)$ & Total \\
\hline$\sum$ & Inglés & 126 & 160 & 286 \\
\hline & Castellano & 3 & 2 & 5 \\
\hline & Japonés & 5 & 3 & 8 \\
\hline & Francés & 9 & 0 & 9 \\
\hline & Italiano & 0 & 5 & 5 \\
\hline & Ruso & 22 & 5 & 27 \\
\hline & Portugués & 6 & 3 & 9 \\
\hline & Polaco & 1 & 1 & 2 \\
\hline & Alemán & 4 & 1 & 5 \\
\hline & Noruego & 0 & 1 & 1 \\
\hline & Sueco & 5 & 0 & 5 \\
\hline & Checo & 1 & 0 & 1 \\
\hline & Húngaro & 2 & 0 & 2 \\
\hline & Chino & 1 & 0 & 1 \\
\hline
\end{tabular}

Figura 2. Número de autores/artículo de los trabajos indicados en MEDLINE sobre salud laboral, nutrición y dieta, ordenados según época de estudio.

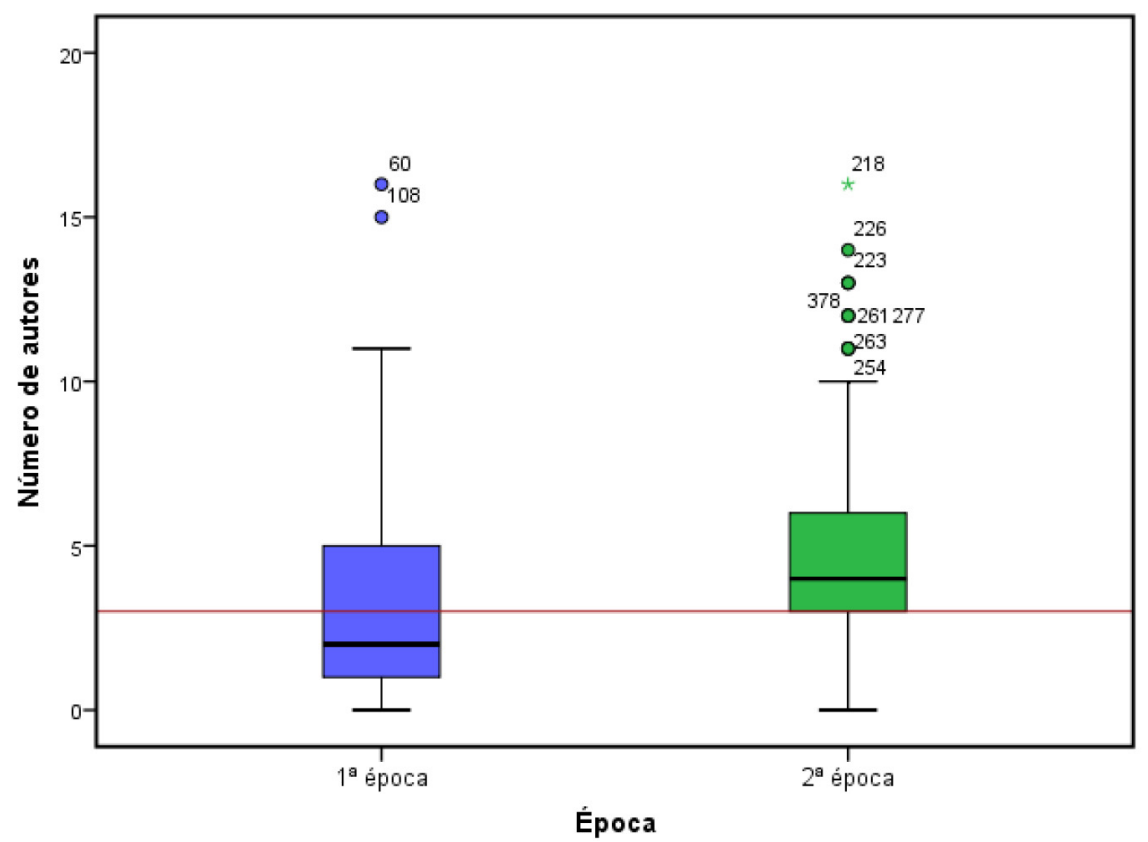




\section{Revistas, impacto y dispersión de la literatura científica}

Se recuperaron un total de 233 revistas que contenían los 386 artículos estudiados, de las que sólo 13 (5,6\%; IC95\% 2,6 - 8,5) publicaron 5 o más artículos. En la tabla III se muestra el listado e impacto de estas publicaciones.

Tabla III. Principales revistas con mayor número de publicaciones y su impacto.

\begin{tabular}{l|c|c|c|c|c|c}
\hline \multicolumn{1}{c|}{ Revista } & f & $\%$ & FI (JCR) & Q (JCR) & SJR & Q (SJR) \\
\hline Journal of Occupational and Environmental Medicine & 17 & 4,4 & 1,36 & 3 & 0,81 & 2 \\
\hline BMC Public Health & 12 & 3,1 & 2,42 & 2 & 1,3 & 1 \\
\hline Occupational Medicine & 8 & 2,1 & - & - & 0,63 & 2 \\
\hline Journal of Agricultural Safety and Health & 7 & 1,8 & - & - & 0,49 & 2 \\
\hline Meditsina truda i promyshlennaia ekologiia & 7 & 1,8 & - & - & 0,11 & 4 \\
\hline Voprosy pitaniia & 7 & 1,8 & - & - & 0,18 & 3 \\
\hline American Journal of Health Promotion & 6 & 1,6 & 2,20 & 2 & 0,81 & 1 \\
\hline Journal of Agromedicine & 6 & 1,6 & 1,32 & 3 & 0,73 & 2 \\
\hline Gigiena i sanitariia & 6 & 1,6 & - & - & 0,2 & 3 \\
\hline Industrial Health & 5 & 1,3 & 1,12 & 4 & 0,56 & 2 \\
\hline American Journal of Industrial Medicine & 5 & 1,3 & 1,73 & 3 & 0,96 & 1 \\
\hline AAOHN journal & 5 & 1,3 & - & - & - & - \\
\hline Business and Health & 5 & 1,3 & - & - & - & - \\
\hline
\end{tabular}

$f=$ Número de artículos publicados; FI(JCR) = Factor de Impacto según la base de datos Journal Citation Report para 2017; $Q(J C R)=$ Cuartil según la mejor posición que ocupa la revista en cualquier categoría en la base de datos Journal Citation Report; $S J R=$ Indicador Scimago Journal Rank para 2017; $Q(S J R)=$ Cuartil según la mejor posición que ocupa la revista en cualquier categoría en la base de datos Scimago Journal \& Country Rank. "." = no consta.

El estudio de la dispersión de la literatura científica recuperada, determina la concentración de una frecuencia similar de artículos en un número diferente de revistas; esta frecuencia de artículos se corresponde aproximadamente con los terciles de producción (33\% de documentos para cada una de las zonas). Así el núcleo principal (zona 1), con 23 revistas $(9,9 \%)$ incluía 128 artículos $(33,2 \%)$; la zona 2 con 81 revistas $(34,8 \%)$ que contenía 129 artículos $(33,4 \%)$ y la zona 3 con 129 revistas $(55,4 \%)$ que englobaban 129 artículos (33,4\%); ver figura 3: gráfico de dispersión según Bradford.

Figura 3. Dispersión en los anillos de Bradford de la producción científica sobre salud laboral, nutrición y dieta recuperada en MEDLINE.

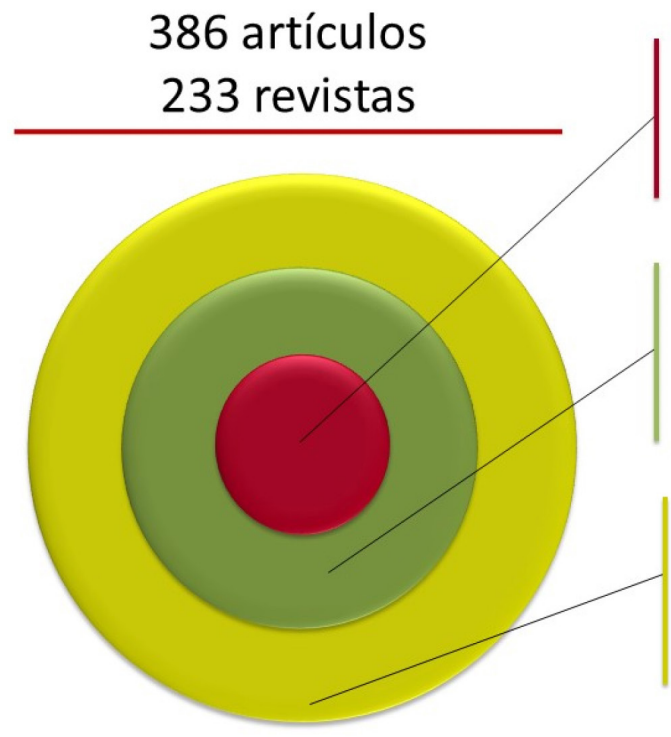

Núcleo

23 revistas $(9,9 \%)$

128 artículos $(33,2 \%)$

\section{Zona 2}

81 revistas $(34,8 \%)$

129 artículos $(33,4 \%)$

\section{Zona 3}

129 revistas $(55,4 \%)$

129 artículos $(33,4 \%)$ 


\section{Acceso al documento primario}

De los 386 artículos a estudio, 253 (65,6\%; IC95\% 60,8 - 70,3) presentaron un enlace activo. La existencia de acceso al texto completo de forma gratuita desde MEDLINE se observó en 126 ocasiones (32,6\%; IC95\% 27,0 - 37,3). Se observaron diferencias significativas, a favor de la $2^{\text {a }}$ época, tanto en el enlace al documento $(90,6 \%$ versus $43,4 \%$; Chi-cuadrado de Pearson $=94,8 ; \mathrm{gl}=1 ; \mathrm{p}<0,001$ ), como a la consulta del texto de forma gratuita (49,7\% versus 17,6\%; Chi-cuadrado de Pearson $=45,2 ; \mathrm{gl}=1 ; \mathrm{p}<0,001)$.

Se constató que 207 documentos (53,6\%) estaban identificados mediante DOI, observando diferencias a favor de la $2^{\text {a }}$ época (82,3\% versus $28,3 \%$; Chi-cuadrado de Pearson $=112,8 ; \mathrm{gl}=1 ; \mathrm{p}<0,001)$.

\section{Clasificación temática de la producción científica}

Para conocer la pertinencia temática de la producción científica a estudio se comprobó que los 386 documentos seleccionados habían sido indizados mediante 531 diferentes Medical Subject Headings (MeSH), pertenecientes a 14 de las 16 áreas temáticas del Thesaurus de la U.S. National Library of Medicine. Los MeSH que fueron utilizados 20 o más veces pueden consultarse en la tabla IV.

Tabla IV. MeSH más frecuentes en la clasificación temática.

\begin{tabular}{l|c|c}
\hline \multicolumn{1}{c|}{ MeSH Major } & Frecuencia & Porcentaje \\
\hline Occupational Health & 246 & 14,2 \\
\hline Health Promotion & 74 & 4,3 \\
\hline Nutritional Status & 37 & 2,1 \\
\hline Occupational Exposure & 34 & 2,0 \\
\hline Diet & 32 & 1,8 \\
\hline Occupational Diseases & 32 & 1,8 \\
\hline Feeding Behavior & 31 & 1,8 \\
\hline Health Behavior & 30 & 1,7 \\
\hline Workplace & 29 & 1,7 \\
\hline Obesity & 27 & 1,6 \\
\hline Breast Feeding & 25 & 1,4 \\
\hline Agriculture & 25 & 1,4 \\
\hline
\end{tabular}

Se constataron diferencias estadísticamente significativas, relacionadas con los Descriptores MeSH utilizados, entre las dos épocas a estudio (Chi-cuadrado de Pearson = 707,5; $\mathrm{gl}=530 ; \mathrm{p}<0,001)$.

Las frecuencias de las 14 grandes áreas temáticas donde se incluyeron los artículos indizados en la base de datos MEDLINE pueden consultarse en la tabla V. Se observaron diferencias en su empleo entre las dos épocas analizadas (Chi-cuadrado de Pearson = $77,7 ; \mathrm{gl}=13 ; \mathrm{p}<0,001)$. 
Tabla V. Frecuencias de las 14 grandes áreas temáticas de los artículos recuperados en la base de datos MEDLINE divididos por época.

\begin{tabular}{l|c|c|c}
\hline \multicolumn{1}{c|}{ Área temática } & $\begin{array}{c}\mathbf{1}^{\mathbf{a}} \text { época } \\
(\mathbf{1 9 4 9}-\mathbf{2 0 0 8})\end{array}$ & $\begin{array}{c}\mathbf{2}^{\mathbf{a}} \text { época } \\
(\mathbf{2 0 0 9}-\mathbf{2 0 1 7})\end{array}$ & Total \\
\hline Health Care & 375 & 344 & 719 \\
\hline Phenomena and Processes & 171 & 198 & 369 \\
\hline Psychiatry and Psychology & 31 & 78 & 109 \\
\hline Persons & 12 & 21 & 33 \\
\hline Organisms & 9 & 15 & 24 \\
\hline Diseases & 100 & 70 & 170 \\
\hline Technology and Food and Beverages & 62 & 58 & 120 \\
\hline Anatomy & 2 & 4 & 6 \\
\hline Information Science & 2 & 3 & 5 \\
\hline Chemicals and Drugs & 60 & 24 & 84 \\
\hline Disciplines and Occupations & 35 & 4 & 39 \\
\hline Analytical, Diagnostic and Therapeutic Techniques and Equipment & 13 & 6 & 19 \\
\hline Anthropology, Education, Sociology and Social Phenomena & 14 & 20 & 34 \\
\hline Humanities & 1 & 3 & 4 \\
\hline
\end{tabular}

\section{DISCUSIÓN}

Este estudio analiza los principales indicadores bibliométricos de la producción científica sobre la salud laboral relacionada con la nutrición, alimentación y dieta indizada en la base de datos bibliográfica MEDLINE, observando que los documentos recuperados están en consonancia con la temática a estudio.

En la evolución de la producción científica se observó un incremento progresivo, aunque sin llegar a ser exponencial, contrariamente a lo expuesto por las teorías cienciométricas que predicen un crecimiento exponencial para periodos superiores a los 30 años $^{8,9}$.

El análisis de la actualidad/obsolescencia, medida tanto por el Índice de Burton Kebler como por el Índice de Price (solo 3 de cada 10 artículos fueron publicados en los últimos 5 años), muestra resultados contrarios y superiores a las tendencias en ciencias de la salud donde prima lo novedoso y reciente ${ }^{10,11}$. Sin embargo, es necesario destacar que entre los años 1965 y 1989 no se indizó ni un solo artículo y que los incluidos en esta base, con fecha anterior a 1990, se deben a indizaciones de los históricos de algunas revistas. Estos datos pueden y deben ayudar a contextualizar la actualidad de esa área temática.

Con respecto a la tipología documental, destaca el predominio de artículos originales y de ensayos clínicos (2 de 3), lógico al tratarse de un área de estudio con aplicación eminentemente empírica y clínica ${ }^{8}$, siendo notable el incremento en los ensayos clínicos en la segunda época, hecho que se puede explicar por la mayor preocupación de instituciones, gobiernos y empresas en aplicar intervenciones para mejorar la salud de los trabajadores ${ }^{12,13}$. El porcentaje de artículos citables es muy superior a los indicadores de evaluación que sugieren una proporción mínima de 1 de cada $2^{14}$. El Índice de Productividad obtenido es similar al observado en otros estudios bibliométricos sobre las ciencias de la nutrición $^{10}$.

En relación al país de procedencia de los artículos, el predominio de la filiación estadounidense es un hecho conocido y recogido ampliamente en la literatura científica ${ }^{15,16}$. A ello contribuye la potencia de sus universidades y la importante financiación pública y privada de sus instituciones y centros de investigación ${ }^{17,18}$. Dato que se relaciona 
íntimamente con el idioma de publicación, donde predominó el inglés, acaparando un porcentaje mayoritario del total de los documentos. Resultados nada novedosos, pues la omnipresencia del inglés es de sobra conocida y una constante en las publicaciones de ciencias de la salud ${ }^{19,20}$. Además, este hecho se ve aumentado debido a la necesidad de los autores e instituciones, de incluir sus publicaciones en las principales bases de datos bibliográficas, como MEDLINE o Web of Science, que pertenecen a instituciones estadounidenses, dado el prestigio que supone su inclusión en ellas ${ }^{21}$. Muestra de lo expuesto anteriormente, es que por continentes, América del Norte acapara casi un tercio de los artículos analizados.

La colaboración entre instituciones fue favorable a la segunda época, la más reciente y posterior a 2008, circunstancia que se explica por la mayor apertura y colaboración del mundo científico y académico aprovechando el gran desarrollo de las tecnologías de la información y comunicación ${ }^{8}$. Aunque los estudios realizados en colaboración entre dos o más centros fueron limitados, uno de cada diez, predominando pequeños grupos que trabajan de manera aislada. Resultados similares a los observados en otras áreas de ciencias de la salud ${ }^{4,10}$. Y, al igual que otras áreas temáticas, fueron escasos los grandes productores $^{20}$.

El número de autores y el índice de cooperación presentaron datos similares y en algunos casos superiores al de otras bibliometrías relacionadas con las ciencias de la nutrición $^{10}$ y la salud laboral ${ }^{4,22}$. Cabe destacar que los datos analizados mostraron una inclinación manifiesta hacia las autorías colectivas, hecho aún más marcado en la segunda época, lo cual indica una clara mejoría en la colaboración entre autores y en la formación de grupos de trabajo, elementos indispensables para el desarrollo de la ciencia.

El medio/bajo impacto y posición de gran parte de las revistas que integran el núcleo principal de Bradford junto con la no inclusión en la Journal Citation Report de muchas otras, muestra una idea de la irregularidad del impacto general del tema a estudio. Uno de los motivos radica en que es una temática alejada de los principales focos de la investigación biomédica ${ }^{23,24}$, por lo que no es casual que las principales revistas del tema ocupen posiciones rezagadas en las principales bases de datos de índices bibliométricos (Journal Citation Reports y Scimago Journal \& Country Rank). Además, se trata de un tema al que aún le queda un largo recorrido y que debe experimentar un gran desarrollo, los potenciales beneficios para trabajadores, empresas y gobiernos son de extrema importancia pues tiene implicaciones en la producción, absentismo, salud laboral, costes de oportunidad, gasto sanitario y un largo etcétera ${ }^{25}$. En cualquier caso, sigue siendo una prioridad para autores e instituciones publicar en las principales revistas científicas, pues permite obtener una mayor visibilidad y por ende un mayor número de citas, siendo éstas la mayor influencia que tiene una revista en términos absolutos. Además, la necesidad curricular impuesta por la carrera académico-profesional de los autores, induce a publicar sus trabajos en las principales revistas contenidas en la base Journal Citation Report de la Web of Science ${ }^{10}$.

Se observó un notable acceso al documento primario, mucho más marcado en la segunda época, donde aproximadamente la mitad de los documentos eran accesibles de forma gratuita, mostrando un amplio apoyo a la iniciativa Open Access (iniciativa de acceso abierto a la literatura científica). Queda patente que existe un claro interés de los editores y sus publicaciones en el hecho de que los documentos estén accesibles a través de Internet pues aumenta su "citabilidad" y el impacto de sus publicaciones ${ }^{26}$.

Queda manifiesto el uso e implantación del Digital Object Identifier (DOI), como también había sido observado en un trabajo de reciente publicación ${ }^{27}$. En este sentido, 8 de cada 10 artículos estudiados en la segunda época presentaba este identificador único que permite un acceso rápido al documento primario ${ }^{28}$.

En relación a la clasificación temática, los resultados obtenidos muestran que los términos están claramente relacionados con la temática a estudio. Destacando aquellos artículos que analizaban el modo en que la salud ocupacional y las intervenciones centradas en la dieta (hidratación, nutrición, acceso a alimentos saludables), ejercicio y 
modificación de conductas (sueño, hábitos tóxicos, etc.) podían influir en la mejora de los factores metabólicos y cardiovasculares de los trabajadores, que a su vez influyen en el rendimiento y el bienestar laboral ${ }^{12,29-31}$.

El término más utilizado fue "Occupational Health», tema principal de estudio junto con los relacionados "Nutritional Status" y "Diet». En cuanto a las áreas temáticas, en ambas épocas destacan "Health Care» y "Phenomena and Processes".

Como posibles limitaciones, indicar que la filiación se empezó a incluir en la base de datos MEDLINE desde el año 1988, por tanto no se pudo disponer de la filiación de todos los documentos. Igualmente, resultó difícil valorar la colaboración institucional con los datos de MEDLINE, ya que es a partir del año 2013 cuando se incluye la institución de todos los firmantes del artículo. No se estudió la relación entre el número de autores por artículo y el número de citas ya que trabajos anteriores refieren una débil relación ${ }^{32}$ o incluso la no existencia de diferencias significativas ${ }^{33}$.

Por todo lo anteriormente expuesto se pudo concluir, que existió relativa obsolescencia, orientación anglófona y filiación estadounidense. Colaboración institucional limitada y destacada de autores. Amplio acceso gratuito al documento primario e impacto irregular de las publicaciones. Notable mejoría de los indicadores bibliométricos a partir del 2008. La clasificación temática cumplió con la materia investigada, siendo un área de conocimiento aún en desarrollo por los potenciales beneficios para trabajadores, empresas y gobiernos.

\section{REFERENCIAS BIBLIOGRÁFICAS}

1. Ramazzini B. Tratado de las enfermedades de los artesanos (reedición). Madrid, España: Escuela Nacional de Medicina del Trabajo del Instituto de Salud Carlos III; 1999.

2. National Institute for Occupational Safety and Health (NIOSH). Total Worker Health [página Web]. Atlanta, USA: US Centers for Disease Control and Prevention; [actualizada 9 de Agosto de 2017; citada 9 en octubre de 2018]. Disponible en: https://www.cdc.gov/spanish/niosh/topics/saludtrabajador.html.

3. Quesada-Risueño P, Sanz Valero J, Wanden-Berghe C. Análisis bibliométrico de la producción científica existente en la base de datos bibliográfica MEDLINE sobre la fibra dietética. Rev Esp Nutr Humana Dietética. 2017;21(1):29-38. DOI: 10.14306/renhyd.21.1.275

4. Sanz-Valero J, Veiga de Cabo J, Rojo-Alonso C, D’Agostino MJ, Wanden-Berghe C, Espulgues Pellicer JX, et al. Los filtros metodológicos: aplicación a la búsqueda bibliográfica en la medicina del trabajo española. Med Segur Trab. 2008;54:75-83.

5. Velázquez López D, Robledillo Colmenares A, Mangas Gallardo I, Veiga-Cabo J, Maqueda Blasco J. Análisis bibliométrico de la revista Medicina y Seguridad del Trabajo durante el periodo 2007-2012. Med Segur Trab. 2013;59:383-92. DOI: 10.4321/S0465-546X2013000400003

6. Castiel LD, Sanz-Valero J. Política científica: manejar la precariedad de los excesos y desnaturalizar la ideología "publicacionista" todopoderosa. Salud Colect. 2009;5(1):5-11.

7. Bordons M, Ángeles Zulueta M. Evaluación de la actividad científica a través de indicadores bibliométricos. Rev Esp Cardiol. 1999;52(10):790-800.

8. Aleixandre Benavent R, Valderrama Zurián JC, Castellano Gómez M, Simó Meléndez R, Navarro Molina C. Factor de impacto de las revistas médicas españolas. Med Clínica (Barc). 2004;123(18):697-701. DOI: 10.1016/S0025-7753(04)75333-6

9. Ardanuy J. Breve introducción a la bibliometría. Barcelona, España: Universidad de Barcelona; 2012.

10. Sanz-Valero J, Gil Á, Wanden-Berghe C, Martínez de Victoria E. Análisis bibliométrico y temático de la producción científica sobre ácidos grasos omega-3 indizada en las bases de datos internacionales sobre ciencias de la salud. Nutr Hosp. 2012;27(Supl 2):41-8.

11. Wanden-Berghe C, Martín-Rodero H. 25 años de investigación en nutrición y alimentación en el espacio iberoamericano del conocimiento. Nutr Hosp. 2012;27:26-33.

12. Lang J, Cluff L, Payne J, Matson-Koffman D, Hampton J. The Centers for Disease Control and Prevention: Findings from the National Healthy Worksite Program. J Occup Environ Med. 2017;59(7):631-41. DOI: 10.1097/JOM.0000000000001045 
13. McCoy K, Stinson K, Scott K, Tenney L, Newman LS. Health Promotion in Small Business: A Systematic Review of Factors Influencing Adoption and Effectiveness of Worksite Wellness Programs. J Occup Environ Med. 2014;56(6):579-87. DOI: 10.1097/JOM.0000000000000171

14. Sanz-Valero J, Casterá VT, Wanden-Berghe C. Estudio bibliométrico de la producción científica publicada por la Revista Panamericana de Salud Pública. Rev Panam Salud Pública. 2014;35(2):81-8.

15. Arnett JJ. The neglected 95\%: why American psychology needs to become less American. Am Psychol. 2008;63(7):602-14. DOI: 10.1037/0003-066X.63.7.602.

16. Bornmann L, Leydesdorff L. Macro-Indicators of Citation Impacts of Six Prolific Countries: InCites Data and the Statistical Significance of Trends. PLoS One. 2013;8(2):e56768. DOI: 10.1371/journal.pone.0056768

17. Academic Ranking of World Universities [Internet]. Shanghai: Shanghai Jiao Tong University; 2013 [acceso el 10 de octubre de 2018]. Academic Ranking of World Universities 2018. Disponible en: http://www. shanghairanking.com/ARWU2018.html.

18. Hather GJ, Haynes W, Higdon R, Kolker N, Stewart EA, Arzberger P, et al. The United States of America and Scientific Research. PLoS One. 2010;5(8):e12203. DOI: 10.1371/journal.pone.0012203

19. U.S. National Library of Medicine (página en Internet). Bethesda: National Institutes of Health; 2003 (actualizado el 07 de mayo de 2018; acceso el 16 de octubre de 2018). MEDLINE: Number of Citations to English Language Articles; Number of Citations Containing Abstracts. Disponible en: http://www.nlm. nih.gov/bsd/medline_lang_distr.html.

20. Franco-Pérez ÁM, Sanz-Valero J, Wanden-Berghe C, Melian-Fleitas L. La producción científica iberoamericana en ciencias de la nutrición: la indización en PubMed y Google Scholar. Nutr Hosp. 2014;30(5):1165-72. DOI: 10.3305/nh.2014.30.5.7723

21. Cremades Pallas R, Burbano P, Valcárcel de la Iglesia MA, Burillo-Putze G, Martín-Sánchez FJ, Miró Ò. Impacto de la inclusión de artículos escritos en inglés en revistas biomédicas españolas de edición multilingüe. An Sist Sanit Navar. 2013;36:467-70. DOI: 10.4321/S1137-66272013000300011.

22. Alonso Arévalo J, Martín Castilla S, Martín Rodero H. Producción científica española sobre salud laboral indizada en el Índice Médico Español (IME). Med Segur Trab. 2008;54:61-73.

23. Topics \& Issues [Internet]. American Public Health Association. Washington, DC. 2018. Disponible en: https://www.apha.org/topics-and-issues.

24. Merigó JM, Núñez A. Influential journals in health research: a bibliometric study. Glob Health. 2016;12(1):e46. DOI: 10.1186/s12992-016-0186-4

25. Pelletier K. A review and analysis of the clinical and cost-effectiveness studies of comprehensive health promotion and disease management programs at the worksite: Update VIII 2008 to 2010. J Occup Environ Med. 2011;53:1310-31. DOI: 10.1097/JOM.0b013e3182337748

26. Heneberg P. Effects of Print Publication Lag in Dual Format Journals on Scientometric Indicators. PLoS One. 2013;8(4):e59877. DOI: 10.1371/journal.pone.0059877

27. Bernabeu-Martínez M A. Sanz Valero J. Análisis bibliométrico y temático de la producción científica existente en la base de datos bibliográfica MEDLINE sobre medicamentos peligrosos en las Unidades de Hospitalización a Domicilio. Hosp Domic. 2018;2(3):101-15. DOI: 10.22585/hospdomic.v2i3.50

28. Brase J, Lautenschlager M, Sens I. The tenth anniversary of assigning DOI names to scientific data and a five year history of DataCite. D-Lib Mag. 2015;21(1-2). DOI: 10.1045/january2015-brase.

29. Shearer J, Graham TE, Skinner TL. Nutra-ergonomics: influence of nutrition on physical employment standards and the health of workers. Appl Physiol Nutr Metab. 2016;41(6 (Suppl. 2)):S165-74. DOI: 10.1139/ apnm-2015-0531

30. Yoon C, Jung H, Kim Y. Evaluation of an Incentive-Based Obesity Management Program in a Workplace. Int J Occup Saf Ergon. 2011;17(2):147-54. DOI: 10.1080/10803548.2011.11076885

31. Naug HL, Colson NJ, Kundur A, Santha Kumar A, Tucakovic L, Roberts M, et al. Occupational health and metabolic risk factors: A pilot intervention for transport workers. Int J Occup Med Environ Health. 2016;29(4):573-84. DOI: 10.13075/ijomeh.1896.00570

32. Leimu R, Koricheva J. Does Scientific Collaboration Increase the Impact of Ecological Articles? Bioscience. 2005; 55(5): 438-43. DOI: 10.1641/0006-3568(2005)055[0438:DSCITI]2.0.CO;2

33. Hart RL. Collaboration and Article Quality in the Literature of Academic Librarianship. J Acad Librariansh. 2007;33(2):190-5. DOI: 10.1016/j.acalib.2006.12.002

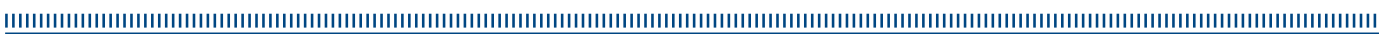

